\title{
Allogeneic mesenchymal stem cells prevent allergic airway inflammation by inducing murine regulatory $T$ cells
}

\author{
H. Kavanagh \& B. P. Mahon \\ Cellular Immunology Laboratory, Institute of Immunology, National University of Ireland Maynooth, Maynooth, Co. Kildare, Ireland
}

To cite this article: Kavanagh $H$, Mahon BP. Allogeneic mesenchymal stem cells prevent allergic airway inflammation by inducing murine regulatory $T$ cells. Allergy 2011; 66: 523-531.

\section{Keywords}

asthma; immune regulation; in vivo;

mesenchymal stem cells; $T_{\text {reg. }}$.

\author{
Correspondence \\ Bernard P. Mahon, Cellular Immunology \\ Laboratory, Institute of Immunology, \\ National University of Ireland Maynooth, \\ Maynooth, Co. Kildare, Ireland. \\ Tel.: +353-1-708-3835 \\ Fax: +353-1-708-3845 \\ E-mail: bp.mahon@nuim.ie
}

Accepted for publication 16 October 2010

DOI:10.1111/j.1398-9995.2010.02509.x

Edited by: Hans-Uwe Simon

\begin{abstract}
Background: Adult bone marrow-derived mesenchymal stem cells (MSC) possess potent immune modulatory effects which support their possible use as a therapy for immune-mediated disease. MSC induce regulatory $\mathrm{T}$ cells $\left(\mathrm{T}_{\mathrm{reg}}\right)$ in vitro although the in vivo relevance of this is not clear.

Objective: This study addressed the hypothesis that adult bone marrow derivedMSC would prevent the pathology associated with allergen-driven airway inflammation, and sought to define the effector mechanism.

Methods: The influence of allogeneic MSC was examined in a model system where $\mathrm{T}_{\text {reg }}$ induction is essential to prevent pathology. This was tested using a combination of a model of ovalbumin-driven inflammation with allogeneic MSC cell therapy.

Results: Systemic administration of allogeneic MSC protected the airways from allergen-induced pathology, reducing airway inflammation and allergen-specific IgE. MSC were not globally suppressive but induced $\mathrm{CD}^{+}{ }^{+} \mathrm{FoxP}^{+} \mathrm{T}$ cells and modulated cell-mediated responses at a local and systemic level, decreasing IL-4 but increasing IL-10 in bronchial fluid and from allergen re-stimulated splenocytes. Moderate dose cyclophosphamide protocols were used to differentially ablate $T_{\text {reg }}$ responses; under these conditions the major beneficial effect of MSC therapy was lost, suggesting induction of $\mathrm{T}_{\text {reg }}$ as the key mechanism of action by MSC in this model. In spite of the elimination of $\mathrm{T}_{\text {reg }}$, a significant reduction in airway eosinophilia persisted in those treated with MSC.

Conclusion: These data demonstrate that MSC induce $\mathrm{T}_{\mathrm{reg}}$ in vivo and reduce allergen-driven pathology. Multiple $\mathrm{T}_{\text {reg }}$ dependent and independent mechanisms of therapeutic action are employed by MSC.
\end{abstract}

Mesenchymal stem or stromal cells (MSC) are a heterogeneous population of cells (1), readily isolated from bone marrow. In addition to potential for tissue repair, MSC possess potent anti-proliferative and anti-inflammatory effects $(2,3)$ which support their therapeutic use for immunemediated diseases. Preclinical models of autoimmune/inflammatory conditions have demonstrated a beneficial role for

\footnotetext{
Abbreviations

BALF, bronchoalveolar lavage fluid; CY, cyclophosphamide; GVHD, graft-versus-host-disease; H\&E, haematoxylin and eosin; MSC, mesenchymal stem cells; OVA, ovalbumin; PAS, periodic acid Schiff; PBS, phosphate buffered saline; PFA, paraformaldehyde; PGE-2, prostaglandin E2; $T_{\text {reg, }}$, regulatory $T$ cells; TGF- $\beta$, transforming growth factor-B.
}

MSC in graft tolerance (4), rheumatoid arthritis (5), and multiple sclerosis (6). Protective roles have also been described in models of acute lung injury, including pulmonary fibrosis (7) and more recently allergic rhinitis (8). However, the precise mechanism by which MSC mediate protection is less clear.

Allergic asthma is an inflammatory disease of the airways, driven in part by Th2- cell induction, eosinophil and mast cell activation and mediator release (9), to establish an inflammatory response combined with profound airway remodeling. Current therapies for allergic asthma include the use of glucocorticoids, however, even long-term pharmacotherapies do not re-program the underlying immune deviation which drives pathology (10). There is an unmet need to develop strategies to reverse immunologic reactivity and chronic airway inflammation. 
Recent advances in immunological understanding have re-evaluated the role of suppression and demonstrated that pathogenic $\mathrm{T}$ cells can be actively countered by regulatory $\mathrm{CD} 4{ }^{+} \mathrm{CD} 25^{+}$FoxP $^{+} \mathrm{T}$ cells $\left(\mathrm{T}_{\text {reg }}\right.$ ) in murine models (11). In these situations the development of allergic airway inflammation is due to inadequate, defective or overwhelmed $\mathrm{T}_{\text {reg }}$ responses. These models provide powerful tools to study the influence of the suppressive or trophic function of MSC. If MSC induction of $\mathrm{CD} 4{ }^{+} \mathrm{CD} 25^{+}$FoxP3 ${ }^{+}$cells in vitro (12, 13 ) is mirrored in vivo, then the ovalbumin (OVA) sensitization model offers a means to test the biological significance of MSC as cell therapeutic inhibitors of allergic airway pathology.

This study addressed the hypothesis that adult bone marrow derived-MSC would prevent the pathology associated with allergen-driven airway inflammation, and sought to define the effector mechanism. Adult bone marrow derived allogeneic MSC actively prevented the induction of allergendriven pathology in a murine model via induction of $\mathrm{T}_{\text {reg }}$ suggesting a novel cell therapy for allergic human disease.

\section{Materials and methods}

\section{Animals}

Allergen sensitization was as previously described (14) using 8- to 12-week old, female BALB/cOlaHsd $\left(\mathrm{H}-2^{\mathrm{d}}\right)$ mice (Harlan, Oxon, UK), whereas FVB/NHanHsd $\left(\mathrm{H}-2^{\mathrm{q}}\right)$ male mice were the source of allogeneic MSC. Mice were maintained according to the regulations of the Irish Department of Health, and the institutional research ethics committee. Mice were sensitized by intra-peritoneal injection of $100 \mu \mathrm{g} /$ $\mathrm{ml}$ ovalbumin (OVA) emulsified in aluminum hydroxide (AlumImject ${ }^{\mathrm{TM}}$ ) (Pierce, IL, USA) on days 0, 7 and 14. Mice were challenged intra-nasally with OVA $(50 \mu \mathrm{g} / \mathrm{ml})$ or sterile PBS (sham) on days 14, 25, 26 and 27 (Fig. 1A).
Isolation and culture of bone marrow derived mesenchymal stem cells

Bone marrow from of $\mathrm{FV} / \mathrm{BN}$ mice was resuspended in Mesencult Basal Medium, supplemented with 10\% (v/v) Mesencult supplement (Stem Cell Technologies, Vancouver, Canada). Cells were maintained as previously described (15), and used between passages 4 and 9 with rigorous purification and quality control to ensure purity as previously described (15). All MSC used were capable of differentiation to the three major mesenchymal lineages (13), and were $\mathrm{MHC}$ class $\mathrm{I}^{+}$, $\mathrm{Sca}^{+}{ }^{+}, \mathrm{CD} 44^{\text {low }}, \mathrm{CD} 106^{\text {low }}, \mathrm{MHC}^{-\mathrm{II}^{-}}, \mathrm{CD} 11 \mathrm{~b}^{-}, \mathrm{CD} 11 \mathrm{c}^{-}$, $\mathrm{CD}^{-} 4^{-}, \mathrm{CD}^{-} 5^{-}$and $\mathrm{CD} 117^{-}$. For paraformaldehyde (PFA)fixed MSC, cells were pelleted and resuspended in $50 \mathrm{ml}$ PFA $(0.5 \%$ in PBS) for $20 \mathrm{~min}$ at room temperature, before extensive washing and use.

\section{MSC therapy}

Allogeneic $\mathrm{H}-2^{\mathrm{q}}$ MSC (or fixed control cells) were washed twice with PBS and resuspended at $5 \times 10^{6}$ cells $/ \mathrm{ml}$. A preliminary investigation was carried out to ascertain whether MSC-induced expansion of $\mathrm{T}_{\text {reg }}$ was dose-dependent and determine the optimum dose. $100 \mu \mathrm{l}$ of MSC at $5 \times 10^{6}$, $5 \times 10^{5}$ or $5 \times 10^{4}$ cells $/ \mathrm{ml}$ were administered i.v. and the expression of FoxP3 by $\mathrm{CD}^{+} \mathrm{T}$ cells was quantified by flow cytometry $0,4,8$ and 12 days following treatment (Fig. $\mathrm{S} 1) . \mathrm{T}_{\text {reg }}$ induction reflected MSC dose and was greatest in mice receiving $0.5 \times 10^{6}$ cells/mouse (Fig. S1); therefore, subsequent experiments utilized this dose. $0.5 \times 10^{6} \mathrm{MSC}$, fixed MSC or PBS were delivered via tail $(100 \mu$ l, i.v. $)$ on days 7 and 14 to $\mathrm{H}-2^{\mathrm{d}}$ mice $(n=8)$ as follows: (i) sham sensitizedPBS alone; (ii) OVA-sensitized mice-PBS; (iii) OVA-sensitized mice-MSC; (iv) OVA-sensitized mice + PFA-fixed MSC; (v) control PBS sham sensitized mice infused with
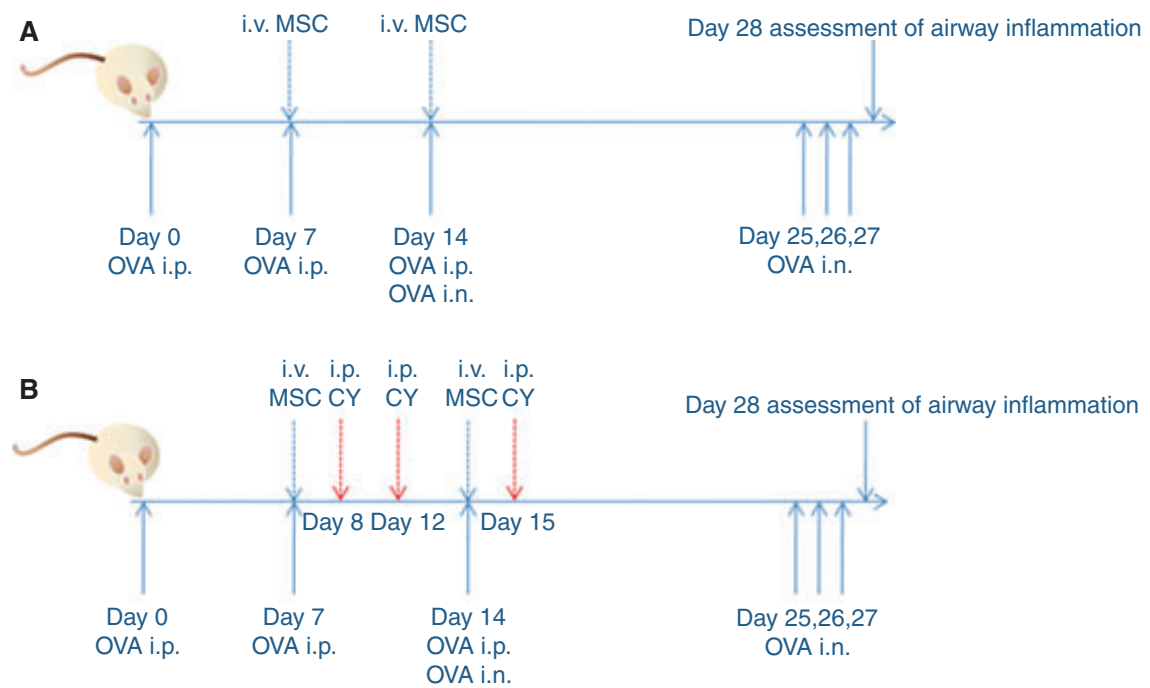

Figure 1 Study sesign. (A) OVA sensitization, (B) CY depletion of $T_{\text {reg. }}$ CY (150 mg/kg) was delivered via i.p. injection on day 8, 12 and 15. 
MSC; (vi) control sham PBS-sensitized with PFA-fixed MSC (Fig. 1A). MSC locating to the airways within $24 \mathrm{~h}$ as previously documented (16) and data not shown. At 28 days, bronchoalveolar lavage fluid, and histopathological studies were performed, serum collected and splenocytes re-stimulated in vitro (14). All experiments were performed at least twice.

\section{Histopathology and airway physiology}

At 28 days BALF was collected (14) and differential cell counts performed as described (14). Nonlavaged lungs were fixed, sectioned and stained with hematoxylin/eosin (H\&E), or combined Discombes/Alcian blue/ periodic acid-Schiff (PAS) stain (14). Pathology was scored using a semi-quantitative scoring system as mild, moderate or severe and shown for convenience in Supporting information (Fig. S2). Lung func- tion was assessed by unrestrained plethysmography and expressed as the surrogate index of enhanced pause $(\mathrm{PenH})$ as previously described (14). This approach has limitations and was used as supporting rather than a definitive indicator of airway hyper-responsiveness.

\section{Measurement of cytokines and antibody response}

IL-4, IL-10 and IL-13 from BALF or antigen re-stimulated or control splenocyte supernatants were analyzed by flow cytometry (Becton-Dickinson, New Jersey, USA), using Cytometric Bead Array Flex Sets (BD Biosciences, Franklin Lakes, NJ, USA) according to manufacturer's instructions. Standard curves and raw data were generated for each cytokine using FCAP Array v1.0.1 software (BD Biosciences). OVA-specific serum IgE was measured by ELISA as previously optimized (17).
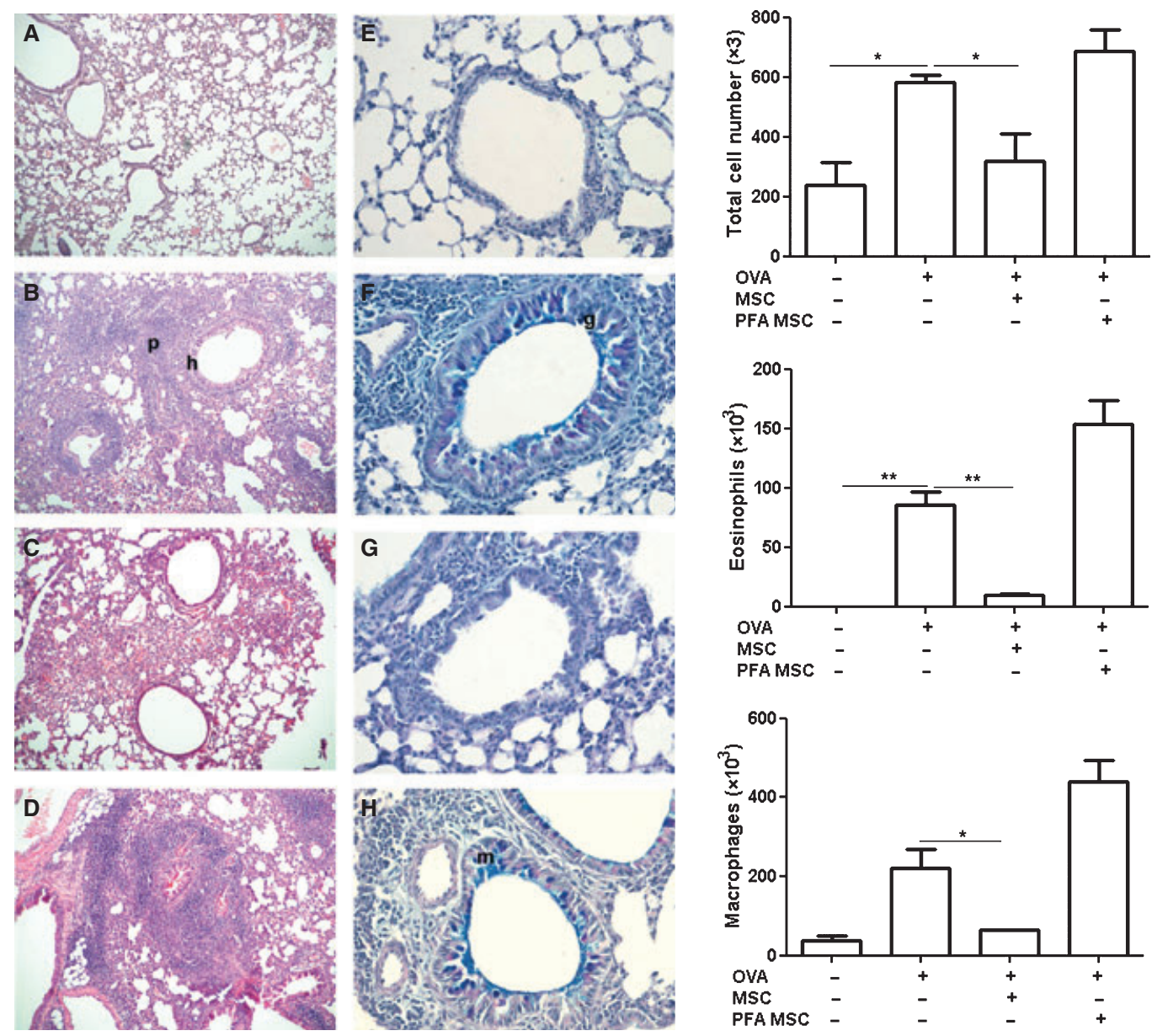

Figure 2 Representative morphological changes in bronchiolar transverse sections of lungs and BAL composition at day 28 from (A \& E) Nonsensitized, (B \& F) OVA-sensitized, (C \& G) OVA-sensitized, MSC treated, (D \& H) OVA-sensitized, PFA-fixed MSC treated. Airway inflammation was detected using H\&E (A-D) (magnification $\times 100)$ and combined Discombes/Alcian blue/PAS $(E-H)$ staining (magnification $\times 400$ ). $\mathbf{p}$ and $\mathbf{h}$ indicate perivascular inflammation and bronchiolar epithelial hypertrophy, respectively. $\mathbf{g}$ and $\mathbf{m}$ indicate goblet cell hyperplasia and mucus secretion, respectively. Negative controls were sham infected/sensitized with saline. The data are representative of three experiments; in each case, at least five animals were assessed. Results are expressed as mean \pm SEM of cell number $\left({ }^{*} P<0.05,{ }^{*} P<0.01\right)$. 


\section{$\mathrm{T}_{\text {reg }}$ depletion and assessment}

In $\mathrm{T}_{\text {reg }}$ studies, mice were sensitized with OVA on day 0,7 and 14, and MSC delivered on days 7 and 14. An established model of pharmacological $T_{\text {reg }}$ depletion was employed (18). Mice received 3 low doses of $150 \mathrm{mg} / \mathrm{kg}$ cyclophosphamide (CY) (Sigma) intraperitoneally on day 8 , 12 and 15 (Fig. 1B). On day 19, splenocytes and lung leukocytes were prepared from representative animals. Single cell suspensions were prepared by dissociating tissue with collagenase D (Sigma) and labeled for surface CD4, CD25 and intracellular FoxP3 as previously described (13). The effectiveness of depletion by dose and time was established in preliminary experiments and verified by the absence of $\mathrm{CD} 4{ }^{+} \mathrm{CD} 25^{+} \mathrm{FoxP}^{+}$populations in spleen or lungs of test animals by flow cytometry at the time points selected (Fig. S3). Remaining mice were challenged with OVA (50 $\mu \mathrm{g}$ in $30 \mu \mathrm{l}$ PBS) intranasally on day 25, 26 and day 27 and assessed as above (Fig. 1B).

\section{Statistical analysis}

Values for all measurements were expressed as the mean \pm standard error of the mean (SEM). Statistical analysis was performed using GRAPHPAD PRISM ${ }^{\text {TM }}$ software (GraphPad, San Diego, CA, USA). Comparison was made using the Kruskal-Wallis test, or the Mann-Whitney test as appropriate. Significance was denoted by $P$-value $<0.05$.

\section{Results}

\section{Allogeneic MSC therapy reduces allergen-driven airway pathology}

The influence of MSC cell therapy was examined in a murine model of allergic pathology. Nonsensitized control mice exhibited no allergen-driven airway inflammation as expected (Fig. 2A), whereas OVA-sensitized mice exhibited typical peribronchial and perivascular inflammation (Fig. 2B). In contrast, MSC therapy resulted in markedly decreased pathology, with decreased peribronchial inflammation (Fig. 2 C). Consistent with these data were physiological observations of a surrogate of airway hyper-responsiveness suggesting that MSC therapy reduced bronchial hyper-reactivity compared to OVA-sensitized mice (Fig. S3). However MSC needed to be viable as delivery of PFA-fixed MSC to allergen sensitized mice resulted in more severe pathology when compared to OVA-sensitized mice, displaying strong perivascular inflammation and bronchiolar epithelial hypertrophy (Fig. 2D). Thus MSC cell therapy reduces classical allergendriven pathology in this model. A consistent feature of asthma is the production of mucus blockage of the peripheral airways (19). MSC delivery reduced airway mucus, whereas PFA-fixed MSC exacerbated goblet cell hyperplasia and mucus secretion in allergen-sensitized mice (Fig. 2E-H). Thus live, but not fixed, MSC therapy reduced multiple characteristic aspects of allergen-driven airway pathology.

\section{Allogeneic MSC therapy protects against allergen-driven lung eosinophilia}

A cardinal feature of allergen-driven airway inflammation is the elevated number of inflammatory cells in the lungs, particularly eosinophils (9). Control mice showed minimal cellularity in bronchoalveolar lavage (Fig. 2), whereas OVA sensitization resulted in significant infiltration $(P<0.05)$. Total cellular infiltration was decreased in OVA-sensitized mice that received MSC, whereas it remained high in sensitized animals treated with PFA-fixed MSC. BALF from control mice had few cells, other than macrophages; however OVA sensitization/challenge resulted in eosinophilic inflammation. Airway eosinophilia was significantly reduced in OVA sensitized mice following MSC delivery $(P<0.05)$, whereas PFA-fixed MSC treatment caused a considerable increase in airway eosinophilia when compared to OVA sensitized mice (Fig. 2). The number of macrophages in BALF was similar to controls. These findings demonstrated that live allogeneic MSC have wide ranging therapeutic influence on allergen-driven airway inflammation and in particular eosinophilic inflammation.

\section{Allogeneic MSC cell therapy induces $\mathrm{T}_{\mathrm{reg}}$ in vivo and modulates allergen-specific immunity}

$\mathrm{IgE}$ induction is a feature of allergen-driven pathologies and OVA sensitization induces $\mathrm{IgE}$ and an allergen-specific Th2 response (20). The capacity for MSC to influence IgE induction was examined by measuring OVA-specific IgE in serum from OVA-sensitized mice in which MSC were used therapeutically. Allogeneic MSC therapy suppressed the allergen-specific IgE response (Fig. 3), in comparison to mice sen-

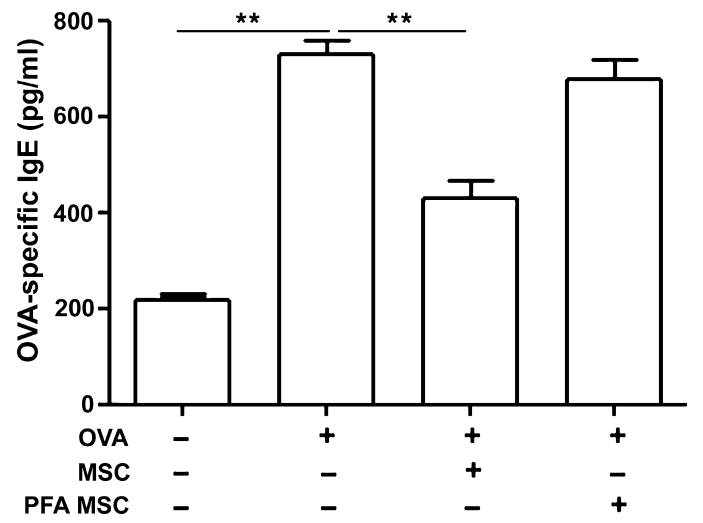

Figure 3 OVA-specific IgE in serum elicited in response to OVA sensitization. Sera were collected on day 28 and OVA-specific serum IgE levels were measured by ELISA. The data are representative of three experiments; in each case, at least five animals were assessed, and each individual assessment was performed independently in triplicate. Concentrations below $200 \mathrm{pg} / \mathrm{ml}$ were considered negative. Results are expressed as mean antibody concentrations \pm SEM $\left({ }^{*} P<0.01,{ }^{* *} P<0.001\right)$. 
A

BALF

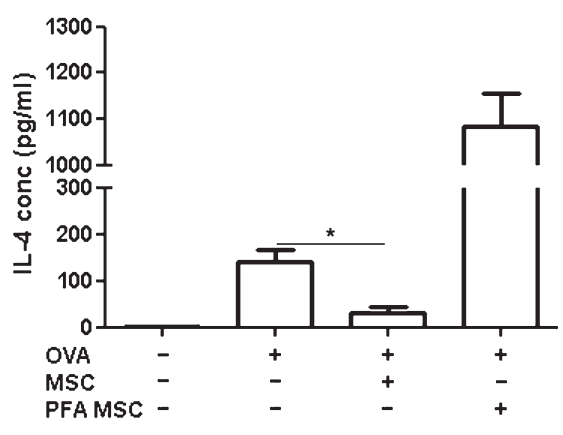

B

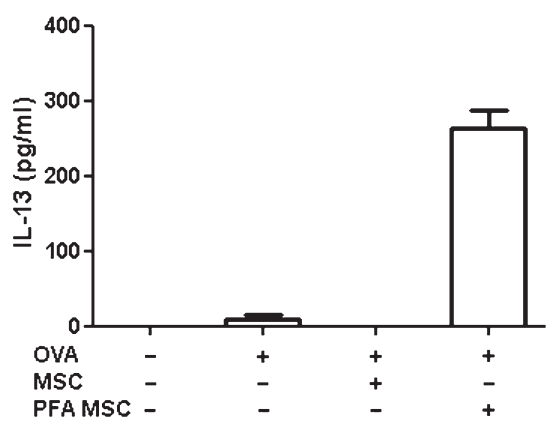

C

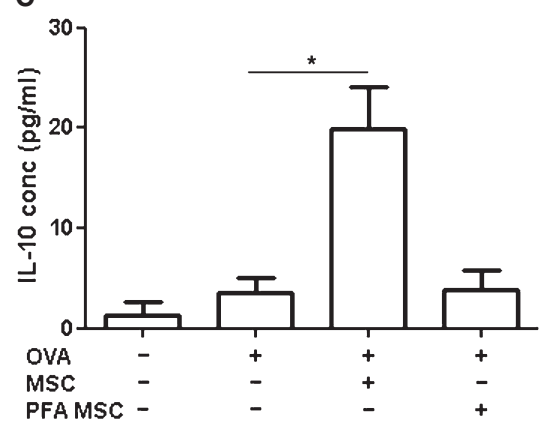

Figure 4 Spleens and BALF were harvested on day 28. Splenocytes were cultured in the presence of media alone $(\square)$ or OVA $(200 \mu \mathrm{g} / \mathrm{ml})(\mathbf{)})$ for $72 \mathrm{~h}$. Cytokine responses from similar cultures are shown for $(A \& C) I L-4,(B \& D) I L-13$ and $(C$ \& E) IL-10.

sitized to OVA alone $(P<0.05)$ (Fig. 3). Sensitized mice that received PFA-fixed MSC showed similar levels of OVA specific IgE to OVA sensitized mice.

The phenomena described above might be explained by MSC-mediated global, nonspecific immune suppression or by MSC interference in immune induction. Therefore, the effect of allogeneic MSC on T cell mediated immunity was examined. Particular attention was given to IL-4 and IL-13 induction as these play well defined roles in allergen-driven pathology (21). As expected, IL-4 and IL-13 in both BALF and splenocyte supernatants were significantly higher in the OVA compared to sham-sensitized mice (Fig. 4). However, a marked reduction in IL-4 and IL-13, but a significant increase in IL-10 (Fig. 4) $(P<0.05)$ was observed in BALF
D

Spleen

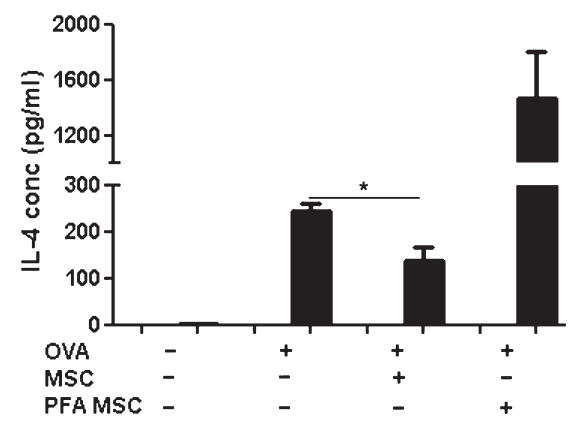

E

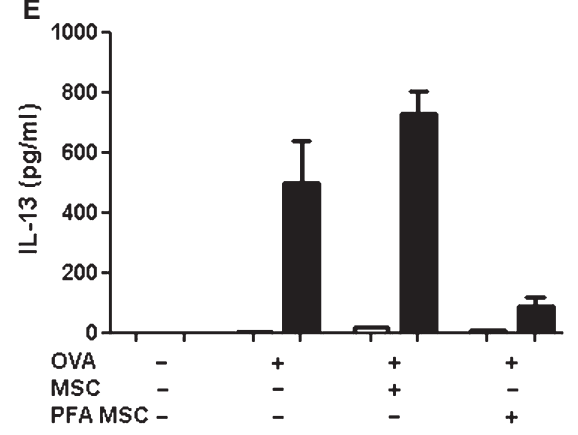

$F$

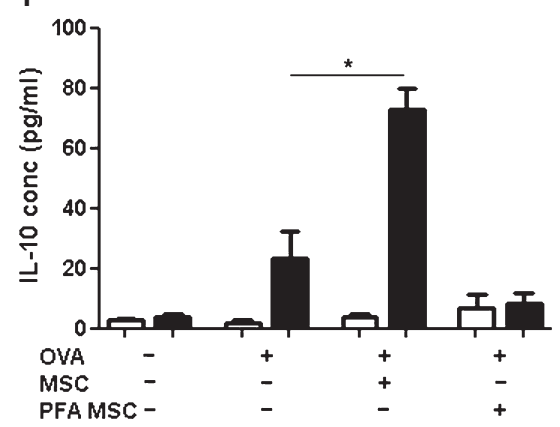

Responses are representative of triplicate experiments, each of which were determined independently from at least nine mice per group and are expressed as means \pm SEM $\left({ }^{*} P<0.05\right)$.

(Fig. 5B) $(P<0.05)$. Similarly, when spleen cells were restimulated ex vivo with allergen, IL-10 was increased but a reduction in IL-13 and IL-4 was observed. This was an important observation as it shows that the protective effect of MSC was a result of targeted, specific modulation of local immunity rather than a global suppression of the immune response.

The induction of local and systemic IL-10 strongly suggested that MSC were inducing a regulatory $\mathrm{T}$ cell population. Therefore, the generation and expansion of $\mathrm{T}_{\text {reg }}$ subsets was assessed in the lungs and spleens of OVA-sensitized mice, and mice that received MSC therapy. MSC induced or expanded a population of regulatory cells; most notably a $\mathrm{CD}^{+}{ }^{+} \mathrm{FoxP}^{+}$population was observed in both the lungs 

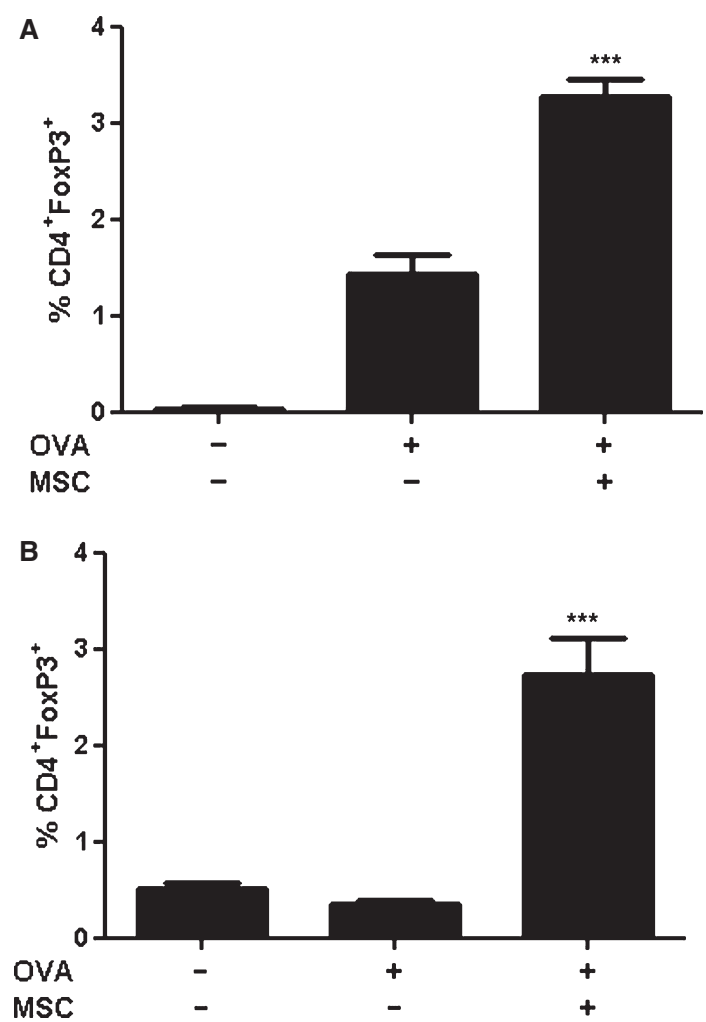

Figure 5 Expression of $\mathrm{CD}^{+} \mathrm{FoxP}^{+}$in lymphoid cells isolated from the lungs (A) and spleen (B) of either OVA-sensitized or OVAsensitized MSC-treated mice. Cells were intracellularly stained with PE-conjugated anti-FoxP3. Responses are representative of duplicate experiments, each of which was determined independently from at least four mice per group.

and spleen from sensitized, MSC-treated mice (Fig. 5). A significant increase in FoxP3 expression $(P<0.05)$ was observed in $\mathrm{CD}^{+} \mathrm{T}$ cells from MSC-treated compared to untreated sensitized mice providing evidence that MSC induce $\mathrm{T}_{\text {reg }}$ populations in vivo.

\section{Regulatory $\mathrm{T}$ cells are required for MSC mediated inhibition of allergic airway inflammation}

Detection of $\mathrm{T}_{\text {reg }}$ does not necessarily equate to an essential function. To investigate whether MSC exerted their immunosuppressive function via induction of $\mathrm{T}_{\text {reg }}$, these suppressor cells were depleted and the effect of MSC delivery on airway pathology was examined. Pharmacological depletion based on cyclophosphamide (CY) administration has been widely used to examine the effect of $\mathrm{T}_{\text {reg }}$ depletion in disease models (22) as it both impairs functionality and depletes $\mathrm{T}_{\text {reg }}$ in vivo (23). Fortunately MSC are $\mathrm{ALDH}^{+}$(24) and thus, resistant to $\mathrm{CY}$ (25), and showed no impairment in differentiation capacity to CY during in vitro exposure (data not shown). A protocol consistent with previous studies (18) was chosen to allow OVA-specific effector cell induction but which depleted $\mathrm{T}_{\text {reg }}$ (Fig. 1B). Contrary to earlier findings, MSC did not con- fer protection when $\mathrm{T}_{\text {reg }}$ were depleted. In the absence of $\mathrm{T}_{\text {reg, }}$, MSC-treated OVA-sensitized mice displayed significant cellular infiltration including peribronchial inflammation at day 28 (Fig. 6) comparable to allergen sensitized mice. Delivery of MSC in the absence of $\mathrm{T}_{\text {reg }}$ also resulted in more pronounced levels of mucus production with some airways showing profound obstruction (Fig. 6). When mice were depleted of $\mathrm{T}_{\text {reg, }}$, there was no observable difference in $\operatorname{IgE}$ between those that received MSC and positive controls (Fig. 6). These data support a model where atopic responses are moderated in vivo by the suppressive influence of constitutive $T_{\text {reg, }}$, and more importantly that $T_{\text {reg }}$ were required for the MSC-mediated reduction of pathology and allergen specific IgE.

MSC cell therapy in $T_{\text {reg-depleted allergen sensitized mice }}$ did not alter the Th2 profile (of IL-4 or -13) in BALF (Fig. 6) and did not elevate IL-10, in direct contrast to when $\mathrm{T}_{\text {reg }}$ were not depleted (Fig. 4). Similarly, MSC therapy in

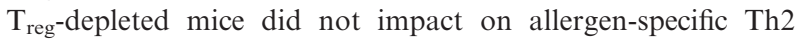
responses in the spleen. Taken together these data strongly suggest that the mechanism of beneficial action by MSC in this model is dependent on the induction of $T_{\text {reg. Thus MSC }}$ modulate allergen specific local and systemic immunity through a $T_{\text {reg }}$ mechanism. A feature of CY treatment in this model is enhanced pathology and increased airway eosinophilia (18). Similar findings are reported here (Fig. 6). However, MSC treatment significantly reduced eosinophilia even in the absence of $T_{\text {reg }}$ (Fig. 6), but did not influence other indices or improve overall pathology. Together these data indicate that whilst $\mathrm{T}_{\text {reg }}$ induction is required to moderate Th2-driven inflammation, an alternative $T_{\text {reg }}$ independent mechanism might also be employed by MSC.

\section{Discussion}

This study demonstrated that adult bone marrow-derived allogeneic MSC actively prevent the induction of allergendriven pathology through a $\mathrm{T}_{\text {reg-dependent mechanism. }}$ Systemic administration of MSC protected the airways from OVA-induced pathology evidenced by reduced lung pathology and cellular inflammation in BALF and reduced allergen specific IgE. MSC were not globally immunosuppressive but rather immunomodulatory, inducing splenic OVA recall responses dominated by IL-10, a cytokine also elevated in BALF by MSC therapy. MSC therapy induced populations of $\mathrm{CD}^{+}{ }^{+} \mathrm{FoxP}^{+} \mathrm{T}$ cells in the lung and spleen. Depletion of $\mathrm{T}_{\text {reg }}$ ablated the protective effect of MSC therapy in terms of the major indices of pathology, and restored class switching to $\mathrm{IgE}$. Thus $\mathrm{T}_{\text {reg }}$ are required for the protective effect of MSC therapy in this model, however MSC continued to affect eosinophilia indicating that MSC also use $\mathrm{T}_{\text {reg-indepen- }}$ dent mechanisms to modulate effector function.

The mechanisms of MSC effector action (and hierarchy) in vivo are poorly understood. MSC can induce direct repair, may be cytoprotective, pro-angiogenic, anti-fibrotic or act through other paracrine effects $(6,7,16)$. We have previously outlined mechanisms by which MSC induce $\mathrm{T}_{\text {reg }}$ in vitro, defining roles for cell contact, TGF- $\beta$ and PGE-2 (13). This 
A
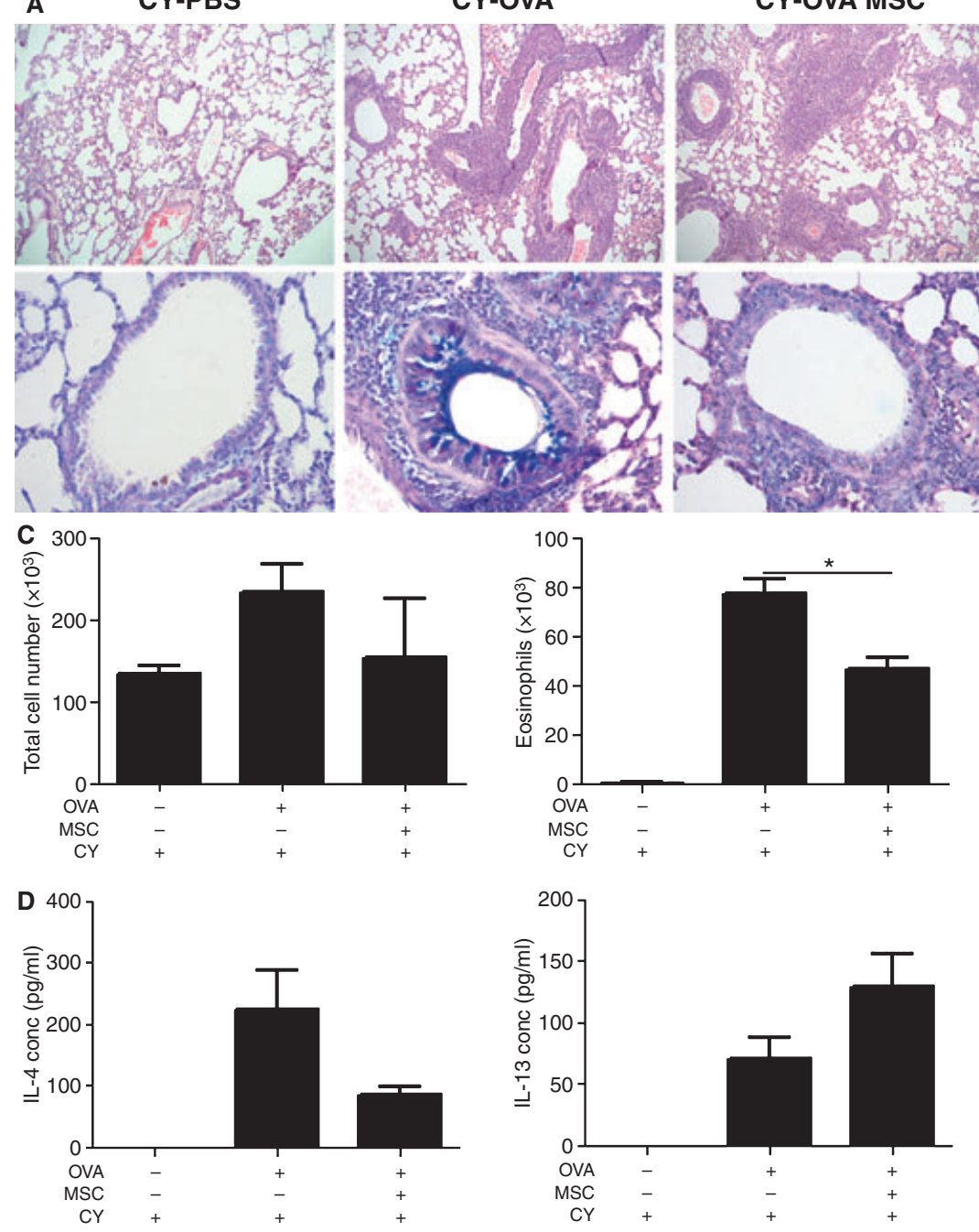

\section{CY-OVA MSC}
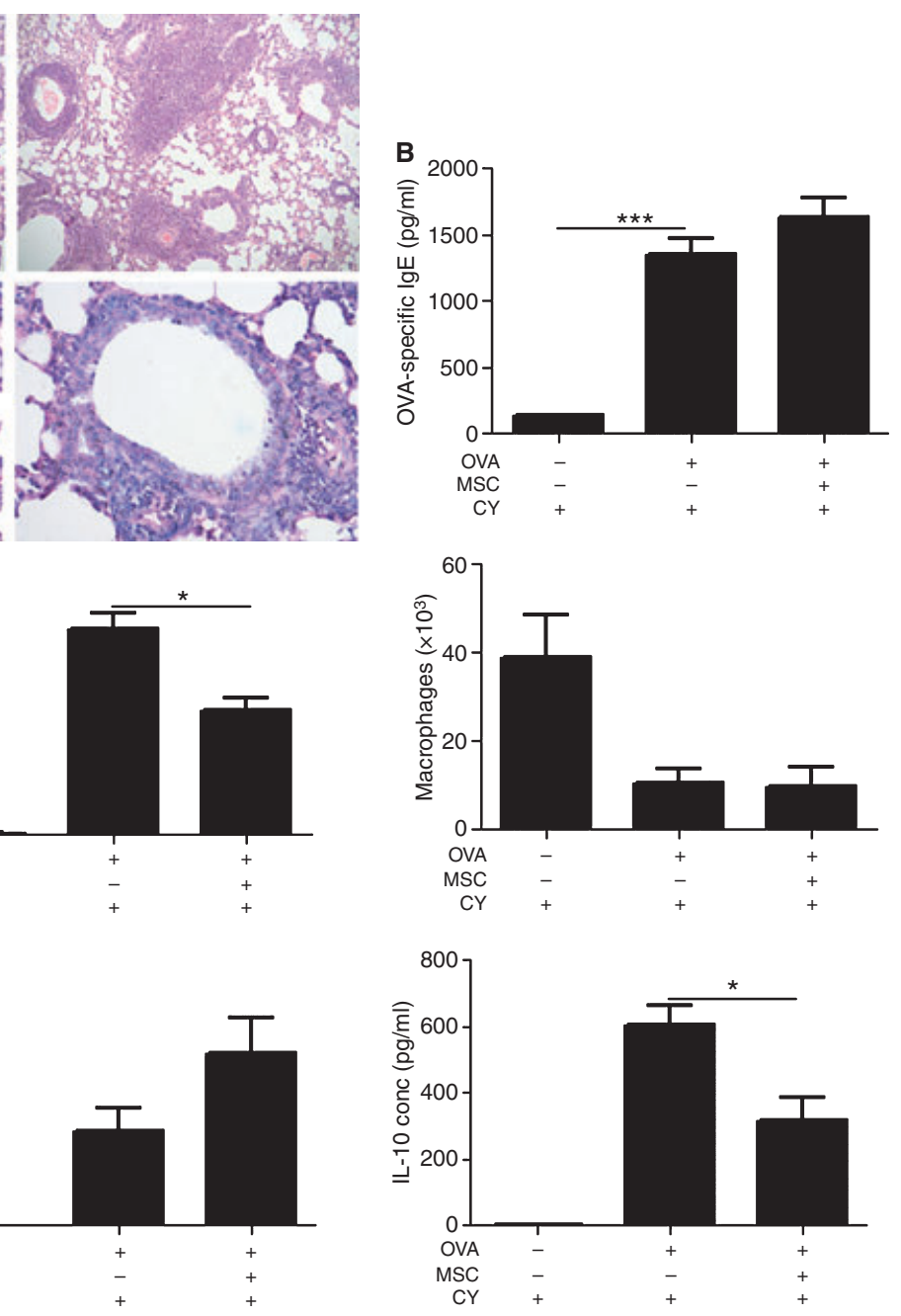

Figure 6 Representative morphological changes at 28 days in bronchiolar transverse sections of lungs from $\mathrm{T}_{\text {reg-depleted, sham- }}$

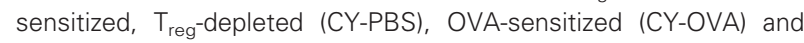

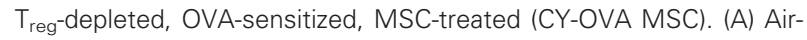
way inflammation detected using haematoxylin and eosin (H\&E) and combined Discombes/Alcian blue/PAS staining of fixed lung

study selected a system where prevention of pathology could be directly linked to $\mathrm{T}_{\text {reg }}$ induction in vivo. Increased $\mathrm{CD}^{+}{ }^{+}$FoxP3 $^{+} \mathrm{T}$ cells and elevated IL-10, locally (lung/ BALF) and systemically (spleen) following MSC therapy was circumstantial evidence for a role for $\mathrm{T}_{\text {reg }}$ as the mechanism for MSC-mediated protection in this model. Although the increase in $\mathrm{FoxP}^{+} \mathrm{T}_{\text {reg }}$ was modest, it is quantitatively similar to recent studies where similar induction was associated with decreased pathology (26). MSC-mediated immunosuppression has been suggested by the expansion of $\mathrm{CD}^{+}{ }^{+} \mathrm{FoxP}^{+}$cells in vitro $(8,26) . \mathrm{CD}^{+}{ }^{+} \mathrm{CD} 25^{+} \mathrm{FoxP}^{+}$ $\mathrm{T}_{\text {reg }}$ are critical for control of antigen-specific inflammation [for review (27)] and their recruitment into the airways can sections. Original magnification, top panels $\times 100$, bottom panels $\times 400$. (B) OVA-specific $\lg E$ in serum elicited in response to OVA sensitization in $T_{\text {reg }}$ depleted mice. (C) Cellular composition of BAL from $T_{\text {reg }}$ depleted mice $24 \mathrm{~h}$ after final OVA exposure. (D) Cytokine profile of BALF elicited by OVA sensitization. All sections are representative of at least three animals.

suppress allergic airway inflammation (28). Recently, IL-10 production by $\mathrm{T}_{\text {reg }}$ was shown to be essential to control immune responses in the lung (29). The current study revealed that MSC therapy increased $\mathrm{CD}^{+}{ }^{+} \mathrm{FoxP}^{+} \mathrm{T}$ cells in lung and spleen which was associated with elevated IL-10 supporting the findings of Rubstov with regard to the importance of $\mathrm{T}_{\text {reg }} / \mathrm{IL}-10$, but more importantly strongly suggesting that MSC induction of $\mathrm{T}_{\text {reg }}$ was not simply an in vitro phenomenon.

$\mathrm{T}_{\text {reg }}$ cell induction in vitro or even in vivo by MSC is an important and interesting finding but its significance depends on the functional contribution to reduced pathology. Here, the contribution of $\mathrm{T}_{\text {reg }}$, induced by MSC, to exert functional 
protection was studied using the cyclophosphamide depletion model. Alternative approaches such as CD25 depletion could not be used as these would interfere with $\mathrm{T}$ cell activation and Th2 induction and confound interpretation, whereas this regime differentially depletes functional $\mathrm{T}_{\text {reg }}$ (30). Using this model it was shown that $\mathrm{T}_{\text {reg }}$ induced by allogeneic MSC therapy were required for the reduction in pathologic score, mucus secretion and allergen-induced IgE. Thus, this study goes beyond demonstrating $\mathrm{T}_{\text {reg }}$ induction in vivo by MSC to show a biological significance for that process.

$\mathrm{T}_{\text {reg }}$ depletion ablated most of the beneficial effects of MSC therapy, indicating the mechanism of protection in this model. However MSC supported a significant reduction in airway eosinophilia despite $\mathrm{T}_{\text {reg }}$ depletion (Fig. 6). This observation was important for two reasons: firstly it indicated that MSC could modulate effector cell function by alternative $\mathrm{T}_{\text {reg-inde- }}$ pendent mechanisms; secondly as reduced eosinophilia is seen here whilst the pathology is impaired, it suggests that altered eosinophilia cannot account for the MSC-mediated effects seen in Fig. 2, and that the primary mechanism by which MSC reduce pathology is via $\mathrm{T}_{\text {reg }}$ induction. We and others have previously shown that MSC express a variety of immunosuppressive cytokines including hepatocyte growth factor (HGF) at concentrations that can suppress allogeneic responses in vitro (31). HGF negatively regulates allergic airway inflammation and hyper-responsiveness (32) via direct attenuation of eosinophil chemotactic function. The expression of HGF by MSC $(31,33)$ and reduced eosinophilia is consistent with such a $T_{\text {reg-independent role, and consistent with }}$ human clinical studies where reduced airway eosinophilia had little impact on pathology (34). The implication of an alternative MSC mechanism of action is that MSC therapy may allow a targeting of complex multi-factorial diseases that involves both fibrotic and inflammatory processes and $\mathrm{T}_{\text {reg-dependent }}$ and independent aspects. Allogeneic MSC possess specific immunomodulatory properties that target critical pathogenic features for the development of allergic asthma. Here we demonstrate the efficacy of MSC based cell therapy in a well characterized murine model. We also illustrate the mechanism of action by which protection is mediated as a proof of concept for MSC based immunotherapy for a broad range of diseases where chronic inflammation results in pathology.

\section{Acknowledgment}

This study was supported by research funding from the Irish Health Research Board, basic research grant RP-2006-274.

\section{Supporting Information}

Additional Supporting Information may be found in the online version of this article at www.wileyonlinelibrary.com:

Figure S1. OVA sensitized mice received $100 \mu \mathrm{MSC}$ i.v. of $5 \times 10^{3}$ (open), $5 \times 10^{4}$ (horizontal shading) or $5 \times 10^{5}$ (black bar) cells $/ \mathrm{ml}$. On day 4 or 8 following treatment, lungs were harvested and digested with collagenase/DNase I for $1 \mathrm{~h}$ followed by intra/extracellular labelling with PE-conjugated anti-FoxP3/Cy5-conjugated anti-CD4. Results are expressed as mean values \pm SD obtained from groups of at least three mice and are representative of two independent experiments. $* P<0.05 ; * * P<0.005$.

Figure S2. Pathology scoring of H\&E-stained lung sections. Original magnification $\times 100$. Perivascular and peribronchial inflammation was evaluated as (A) mild, (B) moderate, and (C) severe. (A) Mild peribronchial inflammation (center) surrounded by clear alveoli and cross-sectioned bronchioles (bottom left, right). (A) Moderate peribronchial inflammation surrounding bronchiole (top right, bottom right) and moderate alveolar inflammation. (C) Severe perivascular and peribronchial inflammation with bronchial epithelial hypertrophy (center, center right).

Figure S3. The effect of $\mathrm{CY}$ treatment on $\mathrm{CD} 4{ }^{+} \mathrm{FoxP}^{+}$ expression. Mice were given $150 \mathrm{mg} / \mathrm{kg}$ of $\mathrm{CY}$ intraperitoneally at day 0 . Spleen cells were isolated followed by intra/ extracellular labeling of anti-FoxP3-PE/anti-CD4-Cy5 then analyzed by flow cytometry. Results are expressed as mean values $\pm \mathrm{SD}$ obtained from groups of at least three mice and are representative of two independent experiments. $* P<0.05 ; * * P 0.005$.

Figure S4. Airway responsiveness was assessed on day 37 by methacholine induced airflow obstruction from conscious mice using whole-body plethysmography in conjunction with the BioSystem XA software (Buxco Electronics, USA) as previously described (14). (A) Nonsensitized (-O-), OVAsensitized (-口-), OVA-sensitized + MSC-treated (- $\mathbf{-}$-), OVAsensitized + PFA-fixed MSC treated (-0-). Results are expressed as mean enhanced pause $(\mathrm{PenH}) \pm \mathrm{SEM}$. Where no error bars are visible, error bars are shorter than the size of the data point symbol.

Please note: Wiley-Blackwell are not responsible for the content or functionality of any supporting materials supplied by the authors. Any queries (other than missing material) should be directed to the corresponding author for the article.

\section{References}

1. Friedenstein AJ, Chailakhjan RK, Lalykina KS. The development of fibroblast colonies in monolayer cultures of guinea-pig bone marrow and spleen cells. Cell Tissue Kinet 1970;3:393-403.

2. Uccelli A, Pistoia V. Immunoregulatory function of mesenchymal stem cells. Eur $J$ Immunol 2006;36:2566-2573.
3. Barry FP, Murphy M, English K. Immunogenicity of adult mesenchymal stem cells. Stem Cell Dev 2005;14:252-265.

4. Le Blanc K, Rasmusson I, Sundberg B. Treatment of severe acute graft-versus-host disease with third party haploidentical mesenchymal stem cells. Lancet 2004;363: 1439-1441.
5. Augello A, Tasso R, Negrini SM, Cancedda R, Pennesi G. Cell therapy using allogeneic bone marrow mesenchymal stem cells prevents tissue damage in collagen-induced arthritis. Arthritis Rheum 2007;56:1175-1186.

6. Constantin G, Marconi S, Rossi B, Angiari S, Calderan L, Anghileri E et al. Adiposederived mesenchymal stem cells ameliorate 
chronic experimental autoimmune encephalomyelitis. Stem Cells 2009;27:2624-2635.

7. Xu J, Woods C, Mora AL, Joodi R, Brigham KL, Iyer $\mathrm{S}$ et al. Prevention of endotoxin-induced systemic response by bone marrow-derived mesenchymal stem cells in mice. Am J Physiol Lung Cell Mol Physiol 2007;293:131-141.

8. Cho KS, Park HK, Park HY, Jung JS, Jeon S-G, Kim Y-K et al. Immunomodulatory effects of adipose tissue-dervied stem cells in an allergic rhinitis mouse model. Stem Cells 2009;27:259-265.

9. Anderson G. The immunobiology of early asthma. Med J Aust 2002;177:S47-S49.

10. Guilbert TW, Morgan WJ, Zeiger RS. Long-term inhaled corticosteroids in preschool children at high risk of asthma. $N$ Engl J Med 2006;354:1985-1997.

11. Sakaguchi S, Powrie F. Emerging challenges in regulatory $\mathrm{T}$ cell function and biology. Science 2007;317:627-629.

12. Prevosto C, Zancolli M, Canevali P, Raffaella Zocchi M, Poggi A. Generation of $\mathrm{CD} 4+$ or $\mathrm{CD} 8+$ regulatory $\mathrm{T}$ cells upon mesenchymal stem cell-lymphocyte interaction. Haematologica 2007;92:881-888.

13. English K, Ryan J, Tobin L, Murphy MJ, Barry FP, Mahon BP. Cell contact, prostaglandin E2 and transforming growth factor beta 1 play non-redundant roles in human mesenchymal stem cell induction of CD4 + CD25highforkhead box P3 + regulatory T cells. Clin Exp Immunol 2009;156: 149-160.

14. Ennis DP, Cassidy J, Mahon BP. Acellular pertussis vaccine protects against exacerbation of allergic asthma due to Bordetella pertussis in a murine model. Clin Diagn Lab Immunol 2005;12:409-417.

15. English K, Barry F, Mahon BP. Murine mesenchymal stem cells suppress dendritic cell migration, maturation and antigen presentation. Immunol Lett 2008;115:50-58.

16. Ortiz LA, Gambelli F, McBride C, Gaupp D, Baddoo M, Kaminski N et al. Mesenchymal stem cell engraftment in lung in enhanced in response to bleomycin exposure and ameliorates its fibrotic effects. PNAS 2003;100:8407-8411.
17. Morokata T, Ishikawa J, Ida K, Yamada T. C57BL/6 mice are more susceptible to antigen-induced eosinophilia than BALB/c mice, irrespective of systemic $\mathrm{T}$ helper $1 / \mathrm{T}$ helper 2 responses. Immunology 1999;98:345-351.

18. Su Y-C, Rolph M, Cooley M, Sewell W. Cyclophosphamide augments inflammation by reducing immunosuppression in a mouse model of allergic airway disease. $J$ Allergy Clin Immunol 2006;117:635-641.

19. Young HW, Sun CX, Evans CM, Dickey BF, Blackbum MR. A3 adenosine receptor signaling contributes to airway mucin secretion after allergen challenge. Am $J$ Respir Cell Mol Biol 2006;35:549-558.

20. Hamelmann E, Takeda K, Oshiba A, Gelfand EW. Role of IgE in the development of allergic airway inflammation and airway hyperresponsiveness-a murine model. Allergy 1999;54:297-305.

21. Romagnani S. Immunologic influences on allergy and the TH1/TH2 balance. $J$ Allergy Clin Immunol [Review] 2004:113:395-400.

22. Uchida S, Susuki K, Akiyama S, Miyamoto M, Juji T, Fujiwara M. Suppressive effect of cyclophosphamide on the progression of lethal graft-versus-host disease in mice-a therapeutic model of fatal post-transfusion GVHD. Ther Immunol 1994;1:313-318.

23. Ikezawa $Y$, Nakazawa M, Tamura C, Takahashi K, Minami M, Ikezawa Z. Cyclophosphamide decrease the number, percentage and the function of $\mathrm{CD} 25+\mathrm{CD} 4+$ regulatory $\mathrm{T}$ cells, which suppress the induction of contact hypersensivity. J Dermatol Sci 2005;39:105-112.

24. Gentry T, Foster S, Winstead L, Deibert E, Fiordalisi M, Balber A. Simultaneous isolation of human BM hematopoietic, endothelial and mesenchymal progenitor cells by flow sorting based on aldehyde dehydrogenase activity: implications for cells therapy. Cytotherapy 2007;9:259-274.

25. Magni M, Shammah S, Schiro R, Mellado W, Dalla-Favera R, Gianni AM. Induction of cyclophosphamide-resistance by aldehydedehydrogenase gene transfer. Blood 1996; 87:1097-1103.

26. Zaiss MA, Frey B, Hess A, Zwerina J, Luther J, Nimmerjahn F et al. (2010)
Regulatory T Cells protect from local and systemic bone destruction in arthritis. J Immunol 2010;7238-7246.

27. Maccario R, Podesta M, Moretta A, Cometa A, Comoli P, Montagna D et al. Interaction of human mesenchymal stem cells with cells involved in alloantigen-specific immune response favors the differentiation of $\mathrm{CD} 4+\mathrm{T}$-cell subsets expressing a regulatory/suppressive phenotype. Haematologica 2005;90:516-525.

28. Tang Q, Bluestone JA. The FoxP3 + regulatory $\mathrm{T}$ cell: a jack of all trades, master of regulation. Nat Immunol 2008;9:239-244.

29. Strickland DH, Stumbles P, Zosky GR. Reversal of airway hyperresponsiveness by induction of airway mucosal $\mathrm{CD} 4{ }^{+} \mathrm{CD} 25^{+}$ regulatory T cells. $J$ Ex Med 2006;203:26492660.

30. Rubtsov Y, Rasmusson J, Chi E. Regulatory T cell-derived interleukin-10 limits inflammation at environmental interfaces. Immunity 2008;28:546-558.

31. Lutziak C, Semnani RT, De Pascalis R, Kashmiri S, Schlom J, Sabzevari H. Inhibition of $\mathrm{CD} 4+\mathrm{CD} 25+\mathrm{T}$ regulatory cell function implicated in enhanced immune response by low-dose cyclophosphamide. Blood 2005;105:2862-2868.

32. Ryan JM, Barry F, Murphy JM, Mahon BP. Interferon-gamma does not break, but promotes the immunosuppressive capacity of adult human mesenchymal stem cells. Exp Immunol 2007;149:353-363.

33. Ito W, Chiba T, Kanehiro A, Kato H, Yamaguchi K, Uehi S et al. Hepatocyte growth factor attenuates eotaxin and PGD2induced chemotaxis of human eosinophils. Allergy 2007;62:415-422.

34. Rehman J, Traktuev D, Li J. Secretion of angiogenic and antiapoptotic factors by human adipose stromal cells. Circulation 2004; 109:1292-1298.

35. Flood-Page PT, Swenson C, Faiferman I, Matthews J, William M, Brannick L et al. A study to evaluate safety and efficacy of mepolizumab in patiens with moderate persistent asthma. Am J Respir Crit Care Med 2007;176:1062-1071. 